\title{
Dynamo effect of spacetime curvature in force-free magnetospheres
}

\author{
Manuel Núñez* \\ Departamento de Análisis Matemático and IMUVA, Universidad de Valladolid, 47005 Valladolid, Spain
}

(Received 8 March 2012; published 23 May 2012)

\begin{abstract}
We study the possibility of growth of the electric and magnetic fields in a force-free plasma due strictly to the gravitational curvature of the spacetime domain where those fields lie. To this end, we identify a total energy by analogy with the results of classical magnetohydrodynamics. After obtaining the general evolution equation for the total energy, we apply to it to the fiducial observers in a number of classical metrics: Schwarzschild, Boyer-Lindquist, Kerr-Schild, Robertson-Walker, and post-Newtonian approximation. As a rule the shift velocity plays the role of minus the fluid velocity in Newtonian MHD, but the details are often highly intricate.
\end{abstract}

DOI: 10.1103/PhysRevD.85.104038

PACS numbers: 04.40.Nr, 47.75.+f, 52.27.Ny, 98.80.Jk

\section{INTRODUCTION}

One of the most intuitive as well as the most relevant phenomena in classical electrodynamics is the growth of magnetic field in an infinitely conducting fluid, or ideal plasma. Magnetic field lines are transported by the flow as material points; as a result, when a large gradient of the velocity stretches the field lines in the appropriate direction, the magnetic energy grows. When the magnetohydrodynamic approximation holds, this may be deduced from the ideal magnetic induction equation

$\frac{\partial \mathbf{B}}{\partial t}=\nabla \times(\mathbf{v} \times \mathbf{B})=-\mathbf{v} \cdot \nabla \mathbf{B}+\mathbf{B} \cdot \nabla \mathbf{v}-\mathbf{B} \nabla \cdot \mathbf{v}$,

where $\mathbf{B}$ is the magnetic field and $\mathbf{v}$ is the fluid velocity. This implies that the density of magnetic energy evolves as

$\frac{1}{2} \frac{\partial B^{2}}{\partial t}=\nabla \cdot\left(-\frac{1}{2} B^{2} \mathbf{v}\right)+\mathbf{B} \cdot \nabla \mathbf{v} \cdot \mathbf{B}-\frac{1}{2} B^{2} \nabla \cdot \mathbf{v}$.

The divergence term accounts for the transport of magnetic field by the flow; the most important source, $\mathbf{B} \cdot \nabla \mathbf{v} \cdot \mathbf{B}$, is the product of the transported field by the original one. The presence of $\nabla \mathbf{v}$ shows that it is the velocity gradient, the key component on the growth of magnetic energy. In fact, if for a finite amount of time the magnetic field points approximately in the direction of an eigenvector of the stress matrix $\nabla \mathbf{v}+{ }^{t} \nabla \mathbf{v}$ with positive eigenvalue, (2) shows an exponential growth of magnetic energy. Obviously, the induction equation should be completed with the momentum equation to take account of the backreaction of the magnetic field upon the flow through the Lorentz force. The study of the so-called dynamo theory constitutes a vast undertaking and it is still far from reaching a full explanation of phenomena such as geomagnetism and sunspots. When the inertial, gravitational, and thermal forces on the plasma are small as compared to the inertia of the electromagnetic field, the Lorentz force will vanish and the plasma will be unable to affect the field; this is called a

*mnjmhd@am.uva.es force-free state. There exists a number of important physical situations where this is valid, notably the solar corona and black hole magnetospheres outside the accretion disk. Another such occurrence happens when the plasma is so tenuous that the density may be taken as zero; since there is no fluid velocity no speak of, this cannot be a classical dynamo. There may exist a magnetic field proceeding from other sources, but the generation of field from empty space does not exist. This, however, does not take into account the source terms of the spacetime curvature, which naturally vanish in a Minkowsky metric. As a result, an observer may find that seed electric or magnetic fields may grow in absence of conducting flow; the fact that this is an observer-dependent phenomenon does not make it less real.

The study of electromagnetism in a relativistic setting is now a well-established discipline. Much of it is already present in the classical monograph [1], but probably the interest on this subject started in earnest with the paper of Blandford and Znajek [2]. Later, McDonald and Thorne settled the basics in $[3,4]$. Today relativistic MHD addresses many subjects, mainly from a numerical viewpoint; one of the most important, black hole ideal electrodynamics, is well understood in force-free magnetospheres $[5,6]$ and extends to the much more complex case of several black holes [7-11]. That gravitational effect may act as a dynamo source even in axisymmetric conditions, in contrast with Cowling's theorem was shown e.g. in $[12,13]$. Generally speaking, numerical treatment of relativistic MHD calls for a $3+1$ decomposition of spacetime [14-16] and an appropriate choice of variables to get the equations in a computationally efficient form [17-20]; it then may be applied to problems other than black holes [21] and the ideal condition may be dropped, although the expression of the resistivity may vary from rather complex [22] to the impossibly complex [23-25].

One of our examples will consider how the motion of a fluid may generate a dynamo source even outside of the fluid body e.g. in a vacuum. We emphasize that we do not deal with the eventual field created by the fluid and 
extending outside it, since the fluid may even be nonconducting; it is only its gravitational effect that may amplify small seed fields. While this effect is quantitatively small for medium-sized astrophysical objects, it is conceptually interesting. The best way to deal with it is to use a postNewtonian approach [26-29].

We will consider the evolution of the electromagnetic energy as viewed from a fiducial observer in a number of well studied instances: Schwarzschild, Boyer-Lindquist, Kerr-Schild, Robertson-Walker, and post-Newtonian setting. The starting equations for MHD are detailed in several of the papers cited before, but probably the simplest form occurs in [30,31], which we will take as our starting point.

\section{II. $3+1$ ELECTRODYNAMICS AND THE MHD INVARIANTS}

In the presentation of relativistic electrodynamics of [30,31], the electric D and magnetic B fields as measured by fiducial observers (FIDOs) are used to define auxiliary three-dimensional fields

$$
\begin{aligned}
& \mathbf{E}=\alpha \mathbf{D}+\boldsymbol{\beta} \times \mathbf{B}, \\
& \mathbf{H}=\alpha \mathbf{B}-\boldsymbol{\beta} \times \mathbf{D},
\end{aligned}
$$

where $\alpha$ is the lapse function and $\beta$ the shift vector in the $3+1$ split of spacetime we are dealing with. (See Appendix A for an explanation of this and subsequent vector identities.) Then the Maxwell equations may be written as

$$
\begin{gathered}
\nabla \cdot \mathbf{B}=0, \\
\frac{1}{\sqrt{\gamma}} \partial_{t}(\sqrt{\gamma} \mathbf{B})+\nabla \times \mathbf{E}=\mathbf{0}, \\
\nabla \cdot \mathbf{D}=\rho, \\
\frac{1}{\sqrt{\gamma}} \partial_{t}(\sqrt{\gamma} \mathbf{D})-\nabla \times \mathbf{H}=\mathbf{J} .
\end{gathered}
$$

Here $\rho$ is the charge density and $\mathbf{J}$ the "absolute" current density. It is related to the current density $\mathbf{j}$ as measured by FIDO by

$$
\mathbf{J}=\alpha \mathbf{j}-\rho \boldsymbol{\beta} .
$$

If we assume a force-free state, the Lorentz force vanishes. This translates as

$$
\rho \mathbf{E}+\mathbf{J} \times \mathbf{B}=\mathbf{0},
$$

or

$$
\rho \mathbf{D}+\mathbf{j} \times \mathbf{B}=\mathbf{0} .
$$

Jointly with (6), this implies

$$
\mathbf{B} \cdot \mathbf{D}=\mathbf{B} \cdot \mathbf{E}=\mathbf{j} \cdot \mathbf{D}=0 .
$$

There are three magnitudes whose integrals in appropriate domains remain invariant in classical MHD [32]. Provided a domain $\Omega$ is closed for the flow and for the field $(\mathbf{v} \cdot \mathbf{n}=$ $\mathbf{B} \cdot \mathbf{n}=0$ at the boundary $\partial \Omega$ ), those are the cross helicity

$$
\mathcal{K}=\frac{1}{2} \int_{\Omega} \mathbf{v} \cdot \mathbf{B} d^{3} \mathbf{x},
$$

and the magnetic helicity

$$
\mathcal{H}=\frac{1}{2} \int_{\Omega} \mathbf{A} \cdot \mathbf{B} d^{3} \mathbf{x},
$$

where $\mathbf{A}$ is a vector potential of $\mathbf{B}, \nabla \times \mathbf{A}=\mathbf{B}$, and the total (kinetic plus magnetic) energy

$$
\mathcal{E}=\frac{1}{2} \int_{\Omega} v^{2}+B^{2} d^{3} \mathbf{x} .
$$

In our case the fluid velocity does not play any role and $\mathcal{K}$ has no meaning. $\mathcal{E}$ should become just the magnetic energy, but in classical MHD the displacement current is taken as zero and the electric field may be ignored, which is no longer the case. Most of this paper is devoted to the evolution of energy, but it is worth to study first the magnetic helicity.

\section{A. Magnetic helicity}

Since $\nabla \cdot \mathbf{B}=0$, provided $\Omega$ is simply connected, there exists a field $\mathbf{A}$ in $\Omega$ such that $\nabla \times \mathbf{A}=\mathbf{B}$, i.e.

$$
B^{i}=\frac{1}{\sqrt{\gamma}} \epsilon^{i j k} \partial_{j} A_{k} .
$$

(See Appendix A.) We may add any gradient to $\mathbf{A}$ with the same result. Equation (6) becomes

$$
\nabla \times\left(\partial_{t} \mathbf{A}+\mathbf{E}\right)=0,
$$

and therefore there exists a scalar field $\Phi$ such that

$$
\partial_{t} \mathbf{A}=-\mathbf{E}+\nabla \Phi .
$$

Since (6) may also be written as

$$
\partial_{t} \mathbf{B}=-\nabla \times \mathbf{E}-\frac{1}{2}\left(\partial_{t} \log \gamma\right) \mathbf{B},
$$

we obtain

$$
\begin{aligned}
\partial_{t}(\mathbf{A} \cdot \mathbf{B})=\partial_{t}\left(A_{i} B^{i}\right)= & \left(-E_{i}+\partial_{i} \Phi\right) B^{i}+A_{i}\left(-(\nabla \times \mathbf{E})^{i}\right. \\
& \left.-\frac{1}{2}\left(\partial_{t} \log \gamma\right) B^{i}\right) \\
= & -\mathbf{E} \cdot \mathbf{B}+\nabla \Phi \cdot \mathbf{B}-\mathbf{A} \cdot(\nabla \times \mathbf{E}) \\
& -\frac{1}{2}\left(\partial_{t} \log \gamma\right) \mathbf{A} \cdot \mathbf{B} .
\end{aligned}
$$

Since $\mathbf{E} \cdot \mathbf{B}=0$ (12), using (A12) with $f=1$,

$$
\nabla \cdot(\mathbf{A} \times \mathbf{E})=\mathbf{B} \cdot \mathbf{E}-\mathbf{A} \cdot(\nabla \times \mathbf{E}),
$$

we get 
$\partial_{t}(\mathbf{A} \cdot \mathbf{B})=\nabla \cdot(\Phi \mathbf{B}+\mathbf{A} \times \mathbf{E})-\frac{1}{2}\left(\partial_{t} \log \gamma\right) \mathbf{A} \cdot \mathbf{B}$.

Let us consider a three-dimensional domain $\Omega$ invariant in time (meaning that the $x^{i}$ coordinates of the points of $\Omega$ do not change in time, although the metric itself may). Assume that $\mathbf{B}$ is always orthogonal to the spatial normal vector at the boundary of $\Omega,\left.\mathbf{B} \cdot \mathbf{n}\right|_{\partial \Omega}=0$. Hodge's theory guarantees that we may choose a vector potential $\mathbf{A}$ such that $\mathbf{A} \times\left.\mathbf{n}\right|_{\partial \Omega}=\mathbf{0}$. Then, for any function $h$,

$$
\begin{aligned}
\frac{\partial}{\partial t} \int_{\Omega} h d V=\frac{\partial}{\partial t} \int_{\Omega} h \sqrt{\gamma} d^{3} \mathbf{x} & =\int_{\Omega} \frac{\partial h}{\partial t} \sqrt{\gamma}+h \frac{\partial \sqrt{\gamma}}{\partial t} d^{3} \mathbf{x} \\
& =\int_{\Omega} \partial_{t} h+\frac{1}{2} h \partial_{t} \log \gamma d V .
\end{aligned}
$$

Thus (22) implies

$$
\begin{aligned}
\frac{\partial \mathcal{H}}{\partial t} & =\int_{\Omega} \nabla \cdot(\Phi \mathbf{B}+\mathbf{A} \times \mathbf{E}) d V \\
& =\int_{\partial \Omega} \Phi \mathbf{B} \cdot \mathbf{n}+(\mathbf{n} \times \mathbf{A}) \cdot \mathbf{E} d \sigma=0 .
\end{aligned}
$$

The same would hold if we take any other vector potential A $+\nabla \psi$, because we would simply add $\psi$ to $\Phi$ in (24). This guarantees the invariance of magnetic helicity even in a curved spacetime. Since helicity is a measure of the knottedness of the magnetic field, this confirms that in a force-free state magnetic field lines do not tend to become more tangled in time.

\section{B. Total energy}

Substituting (3) and (4) in (6) and (8), we obtain

$$
\begin{gathered}
\partial_{t} \mathbf{B}=\boldsymbol{\beta} \cdot \nabla \mathbf{B}-\mathbf{B} \cdot \nabla \boldsymbol{\beta}-\nabla \times(\alpha \mathbf{D})+\eta \mathbf{B}, \\
\partial_{t} \mathbf{D}=\boldsymbol{\beta} \cdot \nabla \mathbf{D}-\mathbf{D} \cdot \nabla \boldsymbol{\beta}+\nabla \times(\alpha \mathbf{B})+\eta \mathbf{D}-\alpha \mathbf{j},
\end{gathered}
$$

where $\eta=\nabla \cdot \boldsymbol{\beta}-\partial_{t} \log \gamma / 2$. Since $D$ and $B$ are the electric and magnetic fields as measured by FIDOs and we want to study their growth, we will study the evolution of $\left(B^{2}+D^{2}\right) / 2$. This is not the same as the electromagnetic energy, whose density is

$$
e=\frac{1}{2}(\mathbf{E} \cdot \mathbf{D}+\mathbf{B} \cdot \mathbf{H})=\frac{1}{2} \alpha\left(B^{2}+D^{2}\right)+\boldsymbol{\beta} \cdot(\mathbf{B} \times \mathbf{D}) .
$$

Both $e=-\alpha T_{t}^{t}$ and the quantity

$$
B^{2}-D^{2}=F_{\mu \nu} F^{\mu \nu},
$$

where $F^{\mu \nu}$ is the Maxwell tensor and $T_{\mu}^{\nu}$ the energymomentum one, are covariant, whereas $\left(B^{2}+D^{2}\right) / 2$ depends on the observer and seems to be an odd quantity to study. Nonetheless, we prefer it to $e$ because the last term in (27), measuring the component of the Poynting vector along the shift velocity, does not occur in classical dynamo theory and we prefer to stay as close as possible to its aims: to study the increase in size of the fields. As for $B^{2}-D^{2}$, it seems an appropriate variable to study, but it has two drawbacks: the first one is that its evolution does not yield a clean equation and it is not easy to interpret the meaning of its terms. The second is that it does not have to remain even positive and thus it is hard to interpret it as a measure of energy. In fact the condition for the magnetohydrodynamic approximation to hold is that the electric field must vanish in the fluid frame [30], and so in any other frame $B^{2}-D^{2}>0$. If we could guarantee e.g. that $B^{2}-D^{2} \geq$ $r B^{2}$ for some positive constant $r$, the growth of $B^{2}+D^{2}$ and the one of $B^{2}-D^{2}$ would mean the same thing. This, however, is not the case. It is shown in [30] that $B^{2}-D^{2}$ may actually turn negative inside the ergosphere of a rotating black hole. Thus, although our results are observer-dependent, they are robust for as long as the magnetohydrodynamic approximation holds true, which is the case we wish to study. Hence we will call $\left(B^{2}+\right.$ $\left.D^{2}\right) / 2$ the density of total energy. Since in what follows we always handle $\mathbf{B} \times \mathbf{D}$, we will call this the Poynting vector, although strictly speaking this is $\mathbf{D} \times \mathbf{B}$.

A simple computation yields

$$
\begin{gathered}
\partial_{t} B^{2}=2\left(\partial_{t} \mathbf{B}\right) \cdot \mathbf{B}+B^{i} B^{j} \partial_{t} \gamma_{i j}, \\
\boldsymbol{\beta} \cdot \nabla B^{2}=2(\boldsymbol{\beta} \cdot \nabla \mathbf{B}) \cdot \mathbf{B}+B^{i} B^{j}\left(\boldsymbol{\beta} \cdot \nabla \gamma_{i j}\right),
\end{gathered}
$$

and the same for D. Using (A12),

$$
\begin{aligned}
- & \nabla \times(\alpha \mathbf{D}) \cdot \mathbf{B}+\nabla \times(\alpha \mathbf{B}) \cdot \mathbf{D} \\
& =2 \nabla \alpha \cdot(\mathbf{B} \times \mathbf{D})+\alpha \nabla \cdot(\mathbf{B} \times \mathbf{D}) .
\end{aligned}
$$

Therefore, multiplying (25) by $\mathbf{B}$, (26) by $\mathbf{D}$, and adding, we obtain the main equation

$$
\begin{aligned}
\frac{1}{2} \partial_{t}\left(B^{2}+D^{2}\right)= & \nabla \cdot\left(\alpha(\mathbf{B} \times \mathbf{D})+\frac{1}{2}\left(B^{2}+D^{2}\right) \boldsymbol{\beta}\right) \\
& +\nabla \alpha \cdot(\mathbf{B} \times \mathbf{D})-\mathbf{B} \cdot \nabla \boldsymbol{\beta} \cdot \mathbf{B}-\mathbf{D} \cdot \nabla \boldsymbol{\beta} \cdot \mathbf{D} \\
& +\frac{1}{2}\left(\nabla \cdot \boldsymbol{\beta}-\partial_{t} \log \gamma\right)\left(B^{2}+D^{2}\right) \\
& +\frac{1}{2}\left(\partial_{t} \gamma_{i j}-\boldsymbol{\beta} \cdot \nabla \gamma_{i j}\right)\left(B^{i} B^{j}+D^{i} D^{j}\right) .
\end{aligned}
$$

Let us look for similarities of (32) and (2). The term within the divergence, as usual, represents the influx or outflux of energy through the boundaries. That means that if we integrate (32) in a three-dimensional domain, there is a net input of energy if the integral of

$$
\alpha(\mathbf{B} \times \mathbf{D})+\frac{1}{2}\left(B^{2}+D^{2}\right) \boldsymbol{\beta},
$$

within $\partial \Omega$ is positive, and an output if negative. The two terms in (33) represent the Poynting vector, which classically represents the flux of electromagnetic energy, plus the total energy density times the shift velocity. When integrating in a domain invariant by $\beta$ (an axisymmetric one in some simple cases), this term disappears.

The true source terms are the remaining ones. Of these, the next one admits an immediate analogy: 


$$
-\mathbf{B} \cdot \nabla \boldsymbol{\beta} \cdot \mathbf{B}-\mathbf{D} \cdot \nabla \boldsymbol{\beta} \cdot \mathbf{D} \sim \mathbf{B} \cdot \nabla \mathbf{v} \cdot \mathbf{B} .
$$

Therefore minus the shift velocity plays the same role as the fluid velocity: whenever the transport of $\mathbf{B}$ (and/or $\mathbf{D}$ ) by $-\beta$ lies roughly in the same direction as the field itself we must expect an increase in total energy In other words, the displacement of spatial coordinates when traveling from one time slice to the next may cause a dynamo effect as viewed by a fiducial observer.

The next analogy is

$$
\frac{1}{2}\left(\nabla \cdot \boldsymbol{\beta}-\partial_{t} \log \gamma\right)\left(B^{2}+D^{2}\right) \sim-B^{2} \nabla \cdot \mathbf{v} .
$$

While the right-hand term vanishes when the fluid is incompressible, the left-hand one is more complex. Nevertheless, if the spatial metric is time-invariant, and $\beta$ is solenoidal and points in the direction of a Killing vector $\left(\partial_{\phi}\right.$ in several axisymmetric cases), then the lefthand term of (35) also vanishes. This, however, does not always occur, as we will see.

Finally, the remaining term

$$
\frac{\alpha}{2}\left(\partial_{t} \gamma_{i j}-\boldsymbol{\beta} \cdot \nabla \gamma_{i j}\right)\left(B^{i} B^{j}+D^{i} D^{j}\right),
$$

has no counterpart in classical MHD, as it represents the proper time derivative of the spatial metric. It measures how the measurement itself varies in time, and it is finite in some simple cases.

Equation (32) shows that with reasonable metrics we cannot expect more than exponential growth of the total energy. That is, if we integrate the density of total energy in a domain $\Omega$ and obtain

$$
\frac{\partial}{\partial t} \int_{\Omega}\left(B^{2}+D^{2}\right) d V \leq M \int_{\Omega}\left(B^{2}+D^{2}\right) d V,
$$

then the total integral energy in $\Omega$ grows at most like $\exp (M t)$. To achieve this we must assume first that the integral of the divergence in (32) is not positive (i.e. there is no inflow on energy). Also, let $\mathbf{w}$ denote the six-component vector $(\mathbf{B} ; \mathbf{D})$, and let $\bar{\gamma}_{i j}$ denote the positive definite bilinear form obtained by duplicating $\gamma_{i j}\left(\bar{\gamma}_{i j} w^{i} w^{j}=B^{2}+\right.$ $D^{2}$ ). Then the remaining terms in (32) may be written as

$$
c_{i j} w^{i} w^{j},
$$

for some coefficients $c_{i j}$ depending on $\nabla \alpha, \nabla \boldsymbol{\beta}, \partial_{t} \log \gamma$, $\partial_{t} \gamma_{i j}-\boldsymbol{\beta} \cdot \nabla \gamma_{i j}$ in a straightforward but complex form. If the bilinear form given in (38) satisfies

$$
\left|c_{i j} w^{i} w^{j}\right| \leq M \bar{\gamma}_{i j} w^{i} w^{j},
$$

for all points within $\Omega$ and all time, the right-hand side of (32) may be bounded by $M\left(B^{2}+D^{2}\right)$. This involves bounding the derivatives of the metric coefficients; although it is essentially a straightforward estimate, the details are messy and a explicit expression is not worth the effort. More interesting would be to show the existence of exponential growth even in the limit of vanishing diffusiv- ity (which is not the same as taking an ideal plasma as we have done). This would imply the existence of a fast dynamo [33], but this subject exceeds our objectives. Instead we will study (32) in a number of physically relevant examples, in order to prove that curvature of spacetime may act as a dynamo in well-known instances.

\section{EXAMPLES OF GRAVITATIONAL DYNAMOS}

In a number of cases the metric coefficients do not depend on time. This may occur because the perturbation of the energy-momentum tensor caused by our timevarying electromagnetic fields is so small as to be safely ignored. Also the shift vector may be directed along a Killing vector, i.e. $\boldsymbol{\beta} \cdot \nabla$ represents the derivative with respect to which all the metric terms are invariant. If in addition $\nabla \cdot \boldsymbol{\beta}=0,(32)$ reduces to

$$
\begin{aligned}
\frac{1}{2} \partial_{t}\left(B^{2}+D^{2}\right)= & \nabla \cdot\left(\alpha(\mathbf{B} \times \mathbf{D})+\frac{1}{2}\left(B^{2}+D^{2}\right) \boldsymbol{\beta}\right) \\
& -\mathbf{B} \cdot \nabla \boldsymbol{\beta} \cdot \mathbf{B}-\mathbf{D} \cdot \nabla \boldsymbol{\beta} \cdot \mathbf{D} .
\end{aligned}
$$

This is the case of several metrics describing spacetime near a stationary black hole.

\section{A. Schwarzschild metric}

It is well-known that the spacetime metric outside a spherical, stationary, uncharged object of mass $m$ is given by

$$
\begin{aligned}
d s^{2}= & -\left(1-\frac{2 m}{r}\right) d t^{2}+\left(1-\frac{2 m}{r}\right)^{-1} d r^{2} \\
& +r^{2}\left(d \theta^{2}+\sin ^{2} \theta d \phi^{2}\right) .
\end{aligned}
$$

Hence

$$
\begin{aligned}
\alpha & =1-\frac{2 m}{r}, \quad \boldsymbol{\beta}=\mathbf{0}, \quad \gamma_{r r}=\left(1-\frac{2 m}{r}\right)^{-1}, \\
\gamma_{\theta \theta} & =r^{2}, \quad \gamma_{\phi \phi}=r^{2} \sin ^{2} \theta .
\end{aligned}
$$

The remaining coefficients vanish. Then (32) becomes

$$
\left(1-\frac{2 m}{r}\right) \frac{1}{2} \partial_{t}\left(B^{2}+D^{2}\right)=\nabla \cdot\left(\left(1-\frac{2 m}{r}\right)^{2}(\mathbf{B} \times \mathbf{D})\right),
$$

i.e.

$$
\frac{1}{2} \partial_{t}\left(B^{2}+D^{2}\right)=\nabla \cdot\left(\left(1-\frac{2 m}{r}\right) \mathbf{B} \times \mathbf{D}\right)+\frac{2 m}{r^{2}}(\mathbf{B} \times \mathbf{D})^{r} .
$$

Thus the variation of total energy within a set $\Omega$ is given by the flux of the Poynting vector times $\alpha$ through its boundary, plus the integral of the radial component of the same vector times $2 m / r^{2}$. No singularity occurs at the horizon $r=2 m$, even if this is set outside the object. Moreover, if one of the boundaries of the domain is set there, it contributes nothing to the boundary integral. This is reasonable 
given that from the viewpoint of the FIDO observer time tends to slow there to zero.

\section{B. Kerr metric in Boyer-Lindquist coordinates}

When the object is rotating with specific angular momentum $a$, the easiest coordinates describing the geometry outside the event horizon are those of Boyer-Lindquist. In this case the FIDO is also a zero angular momentum observer, i.e. it rotates azimuthally with the same constant angular velocity. Taking for simplicity $m=1$, the metric may be written as

$$
\begin{aligned}
d s^{2}= & (z-1) d t^{2}+\frac{\rho^{2}}{\Delta} d r^{2}+\rho^{2} d \theta^{2}+\frac{\Sigma^{2} \sin ^{2} \theta}{\rho^{2}} d \phi^{2} \\
& -2 a z \sin ^{2} \theta d t d \phi,
\end{aligned}
$$

where

$$
\begin{aligned}
& \rho^{2}=r^{2}+a^{2} \cos ^{2} \theta, \quad z=\frac{2 r}{\rho^{2}}, \\
& \Sigma^{2}=\left(r^{2}+a^{2}\right)^{2}-a^{2} \Delta \sin ^{2} \theta, \quad \Delta=r^{2}+a^{2}-2 r .
\end{aligned}
$$

Therefore

$$
\begin{gathered}
\alpha=\frac{\rho}{\Sigma} \sqrt{\Delta}, \\
\beta^{\phi}=-\frac{2 a r}{\Sigma^{2}} .
\end{gathered}
$$

The remaining components are zero. Thus

$$
\nabla \cdot \boldsymbol{\beta}=\partial_{t} \gamma_{i j}=\boldsymbol{\beta} \cdot \nabla \gamma_{i j}=0 .
$$

Let $\mathbf{e}_{\phi}$ denote the unit azimuthal vector. After some manipulation, (32) may be written as

$$
\begin{aligned}
\frac{1}{2} \partial_{t}\left(B^{2}+D^{2}\right)= & \nabla \cdot\left(\frac{\rho}{\Sigma} \sqrt{\Delta}(\mathbf{B} \times \mathbf{D})-\frac{a r}{\Sigma^{2}}\left(B^{2}+D^{2}\right) \mathbf{e}_{\phi}\right) \\
& +\left(\partial_{r}\left(\frac{\rho}{\Sigma} \sqrt{\Delta}\right)\right)(\mathbf{B} \times \mathbf{D})^{r} \\
& +\left(B^{r} B_{\phi}+D^{r} D_{\phi}\right) \partial_{r}\left(\frac{2 a r}{\Sigma^{2}}\right) \\
& +\left(B^{\theta} B_{\phi}+D^{\theta} D_{\phi}\right) \partial_{\theta}\left(\frac{2 a r}{\Sigma^{2}}\right) .
\end{aligned}
$$

While this expression is not so easy to interpret, some things are clear. There is no singularity either at the event horizon $(\Delta=0)$ nor at the simultaneity horizon $(z=1)$. Also the source terms vanish for purely toroidal $\left(B^{r}=\right.$ $\left.B^{\theta}=0\right)$ or poloidal $\left(B^{\phi}=0\right)$ fields, which are commonly used in modelling; only $(\mathbf{B} \times \mathbf{D})^{r}$ acts as a forcing, so at least one of these fields must have an azimuthal component and the other a poloidal one if some dynamo effect occurs.

\section{Kerr metric in Kerr-Schild coordinates}

The Kerr-Schild FIDO rotates with the same angular velocity as the Boyer-Lindquist one but also moves radially towards the center. This avoids the coordinate singularity at the event horizon, but one must pay the price of a complication of the metric. Those coefficients which do not vanish are

$$
\begin{array}{rlrl}
g_{t t} & =z-1, & g_{t \phi} & =-z a \sin ^{2} \theta, \quad g_{t r}=z, \\
g_{r r} & =1+z, & g_{r \phi}=-a(1+z) \sin ^{2} \theta, \quad g_{\theta \theta}=\rho^{2}, \\
g_{\phi \phi} & =\frac{\Sigma^{2} \sin ^{2} \theta}{\rho^{2}} . & &
\end{array}
$$

The terms $z, \rho, \Sigma$ have the same meaning as before. Then

$$
\begin{gathered}
\alpha=\frac{1}{\sqrt{1+z}}=\frac{\rho}{\sqrt{\rho^{2}+2 r}}, \\
\beta^{r}=\frac{z}{1+z}=\frac{2 r}{\rho^{2}+2 r} .
\end{gathered}
$$

The expression of (32) in these coordinates is messy and adds little insight. Some things are worth noticing: although the coefficients are axisymmetric, $\boldsymbol{\beta}$ is radial, not azimuthal as before. Hence in $-\mathbf{B} \cdot \nabla \boldsymbol{\beta} \cdot \mathbf{B}$ the term

$$
-B^{r} B_{r} \frac{\rho}{\sqrt{\rho^{2}+2 r}} \partial_{r}\left(\frac{2 r}{r^{2}+2 r+a^{2} \cos ^{2} \theta}\right)
$$

occurs, which shows when the magnetic or electric field have a radial component, this influences the dynamo. This agrees well with the motion of the Kerr-Schild FIDO. Also $\nabla \cdot \boldsymbol{\beta} \neq 0, \boldsymbol{\beta} \cdot \nabla \gamma_{i j} \neq 0$, so that most of the terms in (32) are finite.

\section{Robertson-Walker type metrics}

We will consider metrics of the type

$$
d s^{2}=-d t^{2}+a(t)^{2} g_{i j} d x^{i} d x^{j},
$$

where $g_{i j}$ does not depend on $t$. The specific RobertsonWalker metric describes a homogeneous and isotropic medium and has the form

$d s^{2}=-d t^{2}+a(t)^{2}\left(\frac{1}{1-k r^{2}} d r^{2}+r^{2} d \theta^{2}+r^{2} \sin ^{2} \theta d \phi^{2}\right)$.

There is no particular advantage in taking this form, so we consider general metrics like (55). For all of them $\alpha=1$, $\boldsymbol{\beta}=\mathbf{0}$. In cosmology $a(t)$ is usually known as the radius of the Universe, as it measures the time evolution of the spatial metric. Thus, if we take as usual

$$
\gamma_{i j}(t)=a(t) g_{i j},
$$

and denote by $\dot{a}$ the time derivative of $a$, by $\dot{\gamma}_{i j}$ the one of $\gamma_{i j}$, (32) reduces to 


$$
\begin{aligned}
\frac{1}{2} \partial_{t}\left(B^{2}+D^{2}\right)= & \nabla \cdot(\mathbf{B} \times \mathbf{D})-\frac{1}{2}\left(\partial_{t} \log \gamma\right)\left(B^{2}+D^{2}\right) \\
& +\frac{1}{2} \dot{\gamma}_{i j}\left(B^{i} B^{j}+D^{i} D^{j}\right) .
\end{aligned}
$$

Since $\dot{\gamma}_{i j}=2(\dot{a} / a) \gamma_{i j}$ and $\gamma=a^{6} g$, we are left with

$$
\partial_{t}\left(B^{2}+D^{2}\right)=2 \nabla \cdot(\mathbf{B} \times \mathbf{D})-2 \frac{\dot{a}}{a}\left(B^{2}+D^{2}\right) .
$$

Consider now a domain $\Omega(t)$ whose spatial coordinates are invariant in time, i.e. it increases or decreases at the same rate as the Universe. Let $\Omega_{0}$ be the three-dimensional domain where these coordinates lie, and assume there is no flux of the Poynting vector through $\partial \Omega$. Then

$$
\begin{aligned}
\partial_{t} \int_{\Omega(t)}\left(B^{2}+D^{2}\right) d V= & \partial_{t} \int_{\Omega_{0}}\left(B^{2}+D^{2}\right) a^{3} \sqrt{g} d^{3} \mathbf{x} \\
= & \int_{\Omega_{0}} \partial_{t}\left(B^{2}+D^{2}\right) a^{3} \sqrt{g} d^{3} \mathbf{x} \\
& +3 a^{2} \dot{a} \int_{\Omega_{0}}\left(B^{2}+D^{2}\right) \sqrt{g} d^{3} \mathbf{x} \\
= & \frac{\dot{a}}{a} \int_{\Omega(t)}\left(B^{2}+D^{2}\right) d V .
\end{aligned}
$$

Hence

$$
\int_{\Omega(t)}\left(B^{2}+D^{2}\right) d V=\frac{a(t)}{a(0)} \int_{\Omega(0)}\left(B^{2}+D^{2}\right) d V .
$$

Thus the total energy within $\Omega$ grows like the radius of the Universe, although the volume of $\Omega$ grows like $a^{3}$. This is probably linked to the fact that the length of the magnetic and electric field lines grows like $a$. Obviously one cannot expect to have force-free plasma in a significant portion of the Universe, but in a region where this holds the very expansion of the Universe has a dynamo effect.

\section{E. Dynamos in the post-Newtonian approximation}

We will consider in greater detail the dynamo effect generated by a fluid motion outside the fluid domain. This could be extended to the motion of a number of point masses instead of a continuous fluid. When the mass is not concentrated enough to generate a black hole, the postNewtonian approximation is often very precise, and as such it has been used to compare general relativity with alternative theories [26]. Naturally the effects e.g. in the Solar System are in the limits of measurability, so we should not expect a large growth of the magnetic field in the vicinity of any astrophysical object due to its gravitational pull; nonetheless the very possibility is interesting, no matter how marginal is the quantitative result. A brief account of the post-Newtonian general relativitistic metric may be found in Appendix B, to which we refer for notation; also a number of cumbersome calculations are there. We will repeat some definitions for convenience. For $\mathbf{x}$ outside $\Omega$, let

$$
\begin{aligned}
w_{i}(\mathbf{x})= & -\frac{7}{2} \int_{\Omega} \frac{\rho\left(\mathbf{x}^{\prime}\right) v_{i}\left(\mathbf{x}^{\prime}\right)}{\left|\mathbf{x}-\mathbf{x}^{\prime}\right|} d^{3} \mathbf{x}^{\prime} \\
& -\frac{1}{2} \int_{\Omega} \frac{\rho\left(\mathbf{x}^{\prime}\right)\left(\mathbf{v}\left(\mathbf{x}^{\prime}\right) \cdot\left(\mathbf{x}-\mathbf{x}^{\prime}\right)\right)\left(x_{i}-x_{i}^{\prime}\right)}{\left|\mathbf{x}-\mathbf{x}^{\prime}\right|^{3}} d^{3} \mathbf{x}^{\prime} .
\end{aligned}
$$

$\rho$ is the density of the fluid and $\mathbf{v}$ its velocity. Then

$$
\begin{aligned}
& \left(-\mathbf{B} \cdot \nabla \boldsymbol{\beta} \cdot \mathbf{B}-\frac{1}{2} B^{i} B^{j}\left(\boldsymbol{\beta} \cdot \nabla \gamma_{i j}\right)+\frac{1}{2}(\nabla \cdot \boldsymbol{\beta}) B^{i} B_{i}\right)(\mathbf{x}) \\
& =\left(-B^{i} B^{j} \partial_{i} w_{j}+\frac{1}{2}|\mathbf{B}|^{2} \operatorname{Divw}\right)(\mathbf{x})+O\left(\delta(\mathbf{x})^{-3}\right)+O\left(\epsilon^{2}\right),
\end{aligned}
$$

where $|\mathbf{B}|^{2}=B^{i} B^{i}$, Divw $=\partial_{i} w_{i}, \delta(\mathbf{x})$ represents the distance of $\mathbf{x}$ to $\Omega$ and $\epsilon$ is the order of magnitude of the main variables with respect to the speed of light. The main term in (63) is expected to behave like $\delta(\mathbf{x})^{-2}$. An identical formula holds for $\mathbf{D}$.

Let $U$ be the Newtonian potential generated by $\rho$, $\Pi$ the internal energy, $p$ the pressure. Since the formulas are already complex enough, we will assume that the fluid motion is stationary; all of these magnitudes are independent of $t$. For all points in $\Omega$, let

$$
G=2 \rho+4 \rho v^{2}+4 \rho U+2 \rho \Pi+6 p .
$$

Then, for points outside $\Omega$,

$\nabla \alpha(\mathbf{x})=\frac{1}{2} \int_{\Omega} \frac{G\left(\mathbf{x}^{\prime}\right)}{\left|\mathbf{x}-\mathbf{x}^{\prime}\right|^{3}}\left(\mathbf{x}-\mathbf{x}^{\prime}\right) d^{3} \mathbf{x}^{\prime}+O\left(\delta(\mathbf{x})^{-3}\right)+O\left(\epsilon^{2}\right)$.

After some calculations on (64), one finds

$$
\begin{aligned}
\left(-B^{i} B^{j} \partial_{i} w_{j}+\frac{1}{2}|\mathbf{B}|^{2} \operatorname{Div} \mathbf{w}\right)(\mathbf{x}) \\
=\int_{\Omega} \frac{\rho\left(\mathbf{x}^{\prime}\right)}{\left|\mathbf{x}-\mathbf{x}^{\prime}\right|^{3}}\left[-3\left(\mathbf{v}\left(\mathbf{x}^{\prime}\right) \cdot \mathbf{B}(\mathbf{x})\right)\left(\left(\mathbf{x}-\mathbf{x}^{\prime}\right) \cdot \mathbf{B}(\mathbf{x})\right)\right. \\
\quad+\frac{3}{2} \mathbf{v}\left(\mathbf{x}^{\prime}\right) \cdot\left(\mathbf{x}-\mathbf{x}^{\prime}\right)|\mathbf{B}(\mathbf{x})|^{2} \\
\left.\quad-\frac{1}{2} \frac{\left.\left(\mathbf{v}\left(\mathbf{x}^{\prime}\right) \cdot\left(\mathbf{x}-\mathbf{x}^{\prime}\right)\right)\left(\left(\mathbf{x}-\mathbf{x}^{\prime}\right) \cdot \mathbf{B}(\mathbf{x})\right)^{2}\right)}{\left|\mathbf{x}-\mathbf{x}^{\prime}\right|^{2}}\right] d^{3} \mathbf{x}^{\prime}
\end{aligned}
$$

This may be written in a simpler form. Let

$$
\mathbf{e}\left(\mathbf{x}, \mathbf{x}^{\prime}\right)=\frac{\mathbf{x}-\mathbf{x}^{\prime}}{\left|\mathbf{x}-\mathbf{x}^{\prime}\right|},
$$

$y_{\|}$the component of the vector $\mathbf{y}$ parallel to $\mathbf{e}, y_{\perp}$ the component orthogonal to it. Then 


$$
\left(-B^{i} B^{j} \partial_{i} w_{j}+\frac{1}{2}|\mathbf{B}|^{2} \operatorname{Divw}\right)(\mathbf{x})=\int_{\Omega} \rho\left(\mathbf{x}^{\prime}\right) \frac{v_{\|}\left(\mathbf{x}^{\prime}\right)\left(-2 B_{\|}(\mathbf{x})^{2}+(3 / 2) B_{\perp}(\mathbf{x})^{2}\right)-3 v_{\perp}\left(\mathbf{x}^{\prime}\right) B_{\perp}(\mathbf{x}) B_{\|}(\mathbf{x})}{\| \mathbf{x}-\left.\mathbf{x}^{\prime}\right|^{2}} d^{3} \mathbf{x}^{\prime} .
$$

The same formula holds for $\mathbf{D}$ instead of $\mathbf{B}$. Let us denote the integral in (68) by $C(\mathbf{B}, \mathbf{x})$. We see that it depends in a complex way of the components of $\mathbf{B}$ (respectfully $\mathbf{D}$ ) parallel or orthogonal to the directional vector e. For the remaining source term, using (65),

$$
\begin{aligned}
\nabla \alpha(\mathbf{x}) \cdot(\mathbf{B} \times \mathbf{D})(\mathbf{x}) & =\frac{1}{2} \int_{\Omega} G\left(\mathbf{x}^{\prime}\right) \frac{\left(\mathbf{x}-\mathbf{x}^{\prime}\right) \cdot(\mathbf{B} \times \mathbf{D})(\mathbf{x})}{\left|\mathbf{x}-\mathbf{x}^{\prime}\right|^{3}} d^{3} \mathbf{x}^{\prime} \\
& =\frac{1}{2} \int_{\Omega} G\left(\mathbf{x}^{\prime}\right) \frac{\left(\mathbf{e}\left(\mathbf{x}, \mathbf{x}^{\prime}\right), \mathbf{B}(\mathbf{x}), \mathbf{D}(\mathbf{x})\right)}{\left|\mathbf{x}-\mathbf{x}^{\prime}\right|^{2}} d^{3} \mathbf{x}^{\prime} .
\end{aligned}
$$

The contribution of this term is positive if the Poynting vector $\mathbf{B} \times \mathbf{D}$ points in the same direction as the outward directional vector $\mathbf{e} ; G$ plays the role of a density of energy in $\Omega$. Let $E(\mathbf{B} \times \mathbf{D})$ denote the integral in (69). For a volume $V$ outside the fluid, far enough from it so that $\delta(\mathbf{x})^{2} \ll \delta(\mathbf{x})^{3}$ for all points in $V$, and ignoring terms of order $O\left(\epsilon^{2}\right)$, (32) yields the approximation

$$
\begin{aligned}
\frac{d}{d t} \frac{1}{2} \int_{V}\left(B^{2}+D^{2}\right) d V \sim & \int_{V} E(\mathbf{B} \times \mathbf{D})(\mathbf{x})+C(\mathbf{B}, \mathbf{x}) \\
& +C(\mathbf{D}, \mathbf{x}) d^{3} \mathbf{x}+\text { boundary terms. }
\end{aligned}
$$

The expression of $E$ is intuitive enough: every point $\mathbf{x}^{\prime}$ in $\Omega$ adds to the dynamo at a point $\mathbf{x}$ when the directional vector $\mathbf{e}$ is aligned with the Poynting vector. The contribution depends on the density $G$ of energy of the fluid at $\mathbf{x}^{\prime}$. However, $C$ is more difficult to interpret, depending as it does on the parallel and perpendicular components of the fields in a complex manner. For a simple case, such a magnetic (or electric) field parallel to $\mathbf{e}$, we are left with $-2 v_{\|} B_{\|}^{2}$; hence, the fluid must go away from $\mathbf{x}$ in order to add to the dynamo. By contrast, if the field is perpendicular to $\mathbf{e}$, we have $(3 / 2) v_{\|} B_{\perp}^{2}$, so the fluid should approach $\mathbf{x}$. It does not seem easy to visualize the actions involved in such an effect.

\section{CONCLUSIONS}

While the study of magnetohydrodynamics in a relativistic context is a vast and well-established discipline, the growth of the magnetic field due to matter motion (the dynamo effect) does not command the same interest. There is little doubt that the main cause which generates dynamos in a classical setting, i.e. the motion of a charged fluid which stretches magnetic field lines, remains valid in any circumstance. However, when the gravitational field is strong enough to modify the curvature of spacetime in a significant way, additional terms acts as sources (or sinks) for the magnetic and electric fields. (The electric field cannot be ignored as it is in classical MHD.) We start from the general relativistic Maxwell equations, which in the form given by Komissarov are simple enough to allow us to use well-known vector identities. Allowing the presence of a conducting fluid would add too many parameters to the problem, so we have restricted ourselves to force-free plasmas. In a sense this is welcome, because it emphasizes the absence of charged fluid motion as the main cause of field growth. We consider the part of electromagnetic energy density due strictly to the size of electric and magnetic fields, and obtain an evolution equation for it. While this quantity is observer-dependent, it agrees better with the spirit of classical MHD and is a robust measure of energy for as long as the magnetohydrodynamic approximation remains valid. It involves in an essential way the lapse function and the shift velocity of the $3+1$ metric, as well as the time derivatives of the metric coefficients. In a sense, minus the shift velocity plays an analogous role to the fluid velocity in classical MHD. To understand better the evolution of these quantities, we study several well-known metrics to see when their fiducial observers would conclude that a dynamo is acting. Those are the Schwarzschild, BoyerLindquist, and Kerr-Schild metrics, which are useful to study force-free fields in the vicinity of a black hole, a group of metric including Robertson-Walker's to see if cosmological growth acts a dynamo, and finally the dynamos generated by a moving (uncharged) fluid outside the body of the fluid. This last example involves the post-Newtonian approximation, and in all probability its real effects would be extremely small; nonetheless, it possesses a theoretical interest. In all these cases, gravitation may act as a dynamo source, although often in a rather involved way.

\section{ACKNOWLEDGMENTS}

The author thanks the anonymous referee for valuable suggestions. This work is partially supported by the Ministry of Science of Spain under Contract No. MTM200912561.

\section{APPENDIX A: VECTOR IDENTITIES}

In the $3+1$ split the spacetime is foliated by spacelike hypersurfaces parameterized by the time coordinate $t$. The metric is written as

$$
d s^{2}=-\alpha^{2} d t^{2}+\gamma_{i j}\left(d x^{i}+\beta^{i} d t\right)\left(d x^{j}+\beta^{j} d t\right),
$$

i.e.

$$
d s^{2}=\left(\beta^{2}-\alpha^{2}\right) d t^{2}+2 \beta_{i} d x^{i} d t+\gamma_{i j} d x^{i} d x^{j},
$$

where $\beta^{i}=\gamma^{i j} \beta_{j}, \beta^{2}=\gamma_{i j} \beta^{i} \beta^{j} . \alpha$ is the so-called lapse function and $\boldsymbol{\beta}$ the shift vector. The local FIDO has four-velocity 


$$
\frac{1}{\alpha}\left(\frac{\partial}{\partial t}-\beta^{i} \frac{\partial}{\partial x^{i}}\right)
$$

and its proper time $\tau$ is related to the universal time $t$ by $d \tau=\alpha d t$. Thus, for any evolving magnitude,

$$
\frac{\partial f}{\partial \tau}=\frac{1}{\alpha}\left(\frac{\partial f}{\partial t}-\boldsymbol{\beta} \cdot \nabla f\right),
$$

which shows that minus the shift velocity $-\boldsymbol{\beta}$ acts as a classical velocity in the lagrangian derivative. This is the source of several dynamo effects.

Classical vector operator act on the spatial coordinates. We will use the following ones:

$\epsilon^{i j k}$ is the Levi-Civita pseudotensor. Its value is 1 when $\{i, j, k\}$ is a positive permutation of $\{1,2,3\},-1$ if negative, 0 if some index repeats. $\epsilon_{i j k}=\epsilon^{i j k}$ is not a tensor. Also

$$
\gamma=\operatorname{det}\left(\gamma_{i j}\right), \quad \xi^{i j k}=\frac{1}{\sqrt{\gamma}} \epsilon^{i j k}, \quad \xi_{i j k}=\sqrt{\gamma} \epsilon_{i j k} .
$$

The cross product (also called the vector product) is

$$
\begin{aligned}
& (\mathbf{u} \times \mathbf{v})_{i}=\xi_{i j k} u^{j} v^{k}=\sqrt{\gamma} \epsilon_{i j k} u^{j} v^{k}, \\
& (\mathbf{u} \times \mathbf{v})^{i}=\xi^{i j k} u_{j} v_{k}=\frac{1}{\sqrt{\gamma}} \epsilon^{i j k} u_{j} v_{k} .
\end{aligned}
$$

Thus

$$
\begin{aligned}
(\mathbf{u} \times \mathbf{v}) \cdot \mathbf{w} & =w_{i}(\mathbf{u} \times \mathbf{v})^{i}=w^{i}(\mathbf{u} \times \mathbf{v})_{i}=\frac{1}{\sqrt{\gamma}} \epsilon^{i j k} w_{i} u_{j} v_{k} \\
& =\sqrt{\gamma} \epsilon_{i j k} w^{i} u^{j} v^{k} .
\end{aligned}
$$

The divergence of $\mathbf{v}$ is denied by

$$
\nabla \cdot \mathbf{v}=\frac{1}{\sqrt{\gamma}} \partial_{i}\left(\sqrt{\gamma} v^{i}\right)
$$

The curl of $\mathbf{v}$ is denied by

$$
(\nabla \times \mathbf{v})^{i}=\xi^{i j k} \partial_{k} v_{j}=\frac{1}{\sqrt{\gamma}} \epsilon^{i j k} \partial_{k} v_{j} .
$$

Thus

$$
\begin{gathered}
\nabla \cdot(\mathbf{u} \times \mathbf{v})=\frac{1}{\sqrt{\gamma}} \partial_{i}\left(\epsilon_{i j k} u^{j} v^{k}\right), \\
\nabla \times(f \mathbf{v})=\nabla f \times \mathbf{v}+f(\nabla \times \mathbf{v}) .
\end{gathered}
$$

Therefore

$$
\begin{array}{r}
-(\nabla \times(f \mathbf{u})) \cdot \mathbf{v}+(\nabla \times(f \mathbf{v})) \cdot \mathbf{u} \\
=2 \nabla f \cdot(\mathbf{v} \times \mathbf{u})+f \nabla \cdot(\mathbf{v} \times \mathbf{u}),
\end{array}
$$

which mimics the Euclidean formula. Finally

$$
\begin{aligned}
(\boldsymbol{\beta} \cdot \nabla \mathbf{v}) \cdot \mathbf{v} & =\frac{1}{2} \beta^{i} \partial_{i}\left(\gamma_{j k} \boldsymbol{v}^{j} \boldsymbol{v}^{k}\right)+\boldsymbol{v}^{j} \boldsymbol{v}^{k} \beta^{i} \partial_{i} \gamma_{j k} \\
& =\frac{1}{2} \boldsymbol{\beta} \cdot \nabla \boldsymbol{v}^{2}+\boldsymbol{v}^{j} \boldsymbol{v}^{k}\left(\boldsymbol{\beta} \cdot \nabla \gamma_{j k}\right) .
\end{aligned}
$$

\section{APPENDIX B: POST-NEWTONIAN APPROXIMATION}

Consider a fluid of density $\rho$ and velocity $\mathbf{v}$ evolving in a subset $\Omega$ of the three-dimensional Euclidean space. Assume that the Newtonian potential $U, v^{2}$, internal energy $\Pi$, and ratio pressure/density $p / \rho$ are of an order $\epsilon$ small as compared with the speed of light 1 . A secondorder approximation, the first one being the Newtonian mechanics, to the metric generated is as follows:

$g_{00}=-1+2 U-2 U^{2}+4 \Phi_{1}+4 \Phi_{2}+2 \Phi_{3}+6 \Phi_{4}+O\left(\epsilon^{3}\right)$,

$$
\begin{gathered}
g_{0 i}=-\frac{7}{2} V_{i}-\frac{1}{2} W_{i}+O\left(\epsilon^{5 / 2}\right), \\
g_{i j}=(1+2 U) \delta_{i j}+O\left(\epsilon^{2}\right),
\end{gathered}
$$

where

$$
\begin{gathered}
U(\mathbf{x})=\int_{\Omega} \frac{\rho\left(\mathbf{x}^{\prime}\right)}{\left|\mathbf{x}-\mathbf{x}^{\prime}\right|} d^{3} \mathbf{x}^{\prime}, \\
\Phi_{1}(\mathbf{x})=\int_{\Omega} \frac{\rho\left(\mathbf{x}^{\prime}\right) v\left(\mathbf{x}^{\prime}\right)^{2}}{\left|\mathbf{x}-\mathbf{x}^{\prime}\right|} d^{3} \mathbf{x}^{\prime}, \\
\Phi_{2}(\mathbf{x})=\int_{\Omega} \frac{\rho\left(\mathbf{x}^{\prime}\right) U\left(\mathbf{x}^{\prime}\right)}{\left|\mathbf{x}-\mathbf{x}^{\prime}\right|} d^{3} \mathbf{x}^{\prime}, \\
\Phi_{3}(\mathbf{x})=\int_{\Omega} \frac{\rho\left(\mathbf{x}^{\prime}\right) \Pi\left(\mathbf{x}^{\prime}\right)}{\left|\mathbf{x}-\mathbf{x}^{\prime}\right|} d^{3} \mathbf{x}^{\prime}, \\
\Phi_{4}(\mathbf{x})=\int_{\Omega} \frac{p\left(\mathbf{x}^{\prime}\right)}{\left|\mathbf{x}-\mathbf{x}^{\prime}\right|} d^{3} \mathbf{x}^{\prime}, \\
V_{i}(\mathbf{x})=\int_{\Omega} \frac{\rho\left(\mathbf{x}^{\prime}\right) v_{i}\left(\mathbf{x}^{\prime}\right)}{\left|\mathbf{x}-\mathbf{x}^{\prime}\right|} d^{3} \mathbf{x}^{\prime}, \\
W_{i}(\mathbf{x})=\int_{\Omega} \frac{\rho\left(\mathbf{x}^{\prime}\right)\left(\mathbf{v}\left(\mathbf{x}^{\prime}\right) \cdot\left(\mathbf{x}-\mathbf{x}^{\prime}\right)\right)\left(x_{i}-x_{i}^{\prime}\right)}{\left|\mathbf{x}-\mathbf{x}^{\prime}\right|^{3}} d^{3} \mathbf{x}^{\prime}
\end{gathered}
$$

Recall that these are integrals with respect to the Euclidean measure, and that all scalar products have Euclidean meanings. To emphasize this point we will write e.g. $|\mathbf{B}|^{2}$ instead of $B^{2}$ for any vector field.

In the $3+1$ presentation of the metric,

$$
\begin{aligned}
\gamma_{i j} & =g_{i j}, \quad \beta^{j}=g^{j i} g_{0 i}, \quad \beta^{2}=g_{i j} \beta^{i} \beta^{j}, \\
\alpha^{2} & =g^{i j} g_{0 i} g_{0 j}-g_{00} .
\end{aligned}
$$

All the integrals above converge if $\rho, \rho v^{2}, \rho \Pi, p$, and $\rho v_{i}$ are Lebesgue integrable in $\Omega$, except for $\Phi_{2}$, for which we need an additional condition, since $U$ occurs in the integrand. If $\rho$ is also square integrable, it is enough. In particular this holds if $\Omega$ is bounded and all the previous 
magnitudes are also bounded in $\Omega$. In this case we may add that they are differentiable, and their derivatives are as follows: if

$$
F(\mathbf{x})=\int_{\Omega} \frac{f\left(\mathbf{x}^{\prime}\right)}{\left|\mathbf{x}-\mathbf{x}^{\prime}\right|} d^{3} \mathbf{x}^{\prime}
$$

then

$$
\partial_{i} F(\mathbf{x})=\int_{\Omega} \frac{f\left(\mathbf{x}^{\prime}\right)}{\left|\mathbf{x}-\mathbf{x}^{\prime}\right|^{3}}\left(x_{i}-x_{i}^{\prime}\right) d^{3} \mathbf{x}^{\prime},
$$

and this integral is also convergent. This covers all the functions in (B4)-(B9), even (B6), since $U$ is bounded. For (B10), we have

$$
\begin{aligned}
\partial_{i} W_{j}(\mathbf{x})= & \int_{\Omega}-\frac{\rho\left(\mathbf{x}^{\prime}\right)\left(\mathbf{v}\left(\mathbf{x}^{\prime}\right) \cdot\left(\mathbf{x}-\mathbf{x}^{\prime}\right)\right)\left(x_{i}-x_{i}^{\prime}\right)\left(x_{j}-x_{j}^{\prime}\right)}{\left|\mathbf{x}-\mathbf{x}^{\prime}\right|^{5}} \\
& +\frac{\rho\left(\mathbf{x}^{\prime}\right) v_{i}\left(\mathbf{x}^{\prime}\right)\left(x_{j}-x_{j}^{\prime}\right)}{\left|\mathbf{x}-\mathbf{x}^{\prime}\right|^{3}} \\
& +\frac{\rho\left(\mathbf{x}^{\prime}\right) \mathbf{v}\left(\mathbf{x}^{\prime}\right) \cdot\left(\mathbf{x}-\mathbf{x}^{\prime}\right)}{\left|\mathbf{x}-\mathbf{x}^{\prime}\right|^{3}} \delta_{i j} d^{3} \mathbf{x}^{\prime} .
\end{aligned}
$$

We see that the functions are divided by terms of the form $\left|\mathbf{x}-\mathbf{x}^{\prime}\right|$, whereas their derivatives are divided by $\left|\mathbf{x}-\mathbf{x}^{\prime}\right|^{2}$, which yields an easy estimate of the decay of these magnitudes for points away from $\Omega$. If we denote by $\delta(\mathbf{x})$ the distance of a point $\mathbf{x}$ to $\Omega$, then there exists a constant $M$ such that for any of the functions $F$ occurring in (B4)-(10),

$$
|F(\mathbf{x})| \leq \frac{M}{\delta(\mathbf{x})}, \quad|\nabla F(\mathbf{x})| \leq \frac{M}{\delta(\mathbf{x})^{2}} .
$$

Notice that although this bound becomes singular when $\mathbf{x}$ approaches $\Omega$, neither the functions $F$ nor $\nabla F$ do.

For our calculations we will ignore the remainders of order $O\left(\epsilon^{2}\right)$, although later we will add them to the final expressions. Let $h=1+2 U, w_{j}=g_{0 j}$. Then

$$
\gamma^{i j}=\frac{1}{h} \delta^{i j}, \quad \boldsymbol{\beta}=\frac{1}{h} \mathbf{w} .
$$

Therefore

$$
\begin{gathered}
\partial_{i} \beta^{j}=-\frac{\partial_{i} h}{h^{2}} w_{j}+\frac{1}{h} \partial_{i} w_{j}, \\
\boldsymbol{\beta} \cdot \nabla \gamma_{i j}=\beta^{k} \partial_{k}\left(h \delta_{i j}\right)=\frac{\mathbf{w} \cdot \nabla h}{h} \delta_{i j}, \\
\nabla \cdot \boldsymbol{\beta}=\frac{1}{\sqrt{g}} \partial_{i}\left(\sqrt{g} \beta^{i}\right)=\frac{1}{h^{3 / 2}} \partial_{i}\left(h^{3 / 2} \frac{w_{i}}{h}\right) \\
=\frac{\mathbf{w} \cdot \nabla h}{2 h^{2}}+\frac{\operatorname{Divw}}{h} .
\end{gathered}
$$

We write Divw instead of $\nabla \cdot \mathbf{w}$ to emphasize that this is the Euclidean divergence $\partial_{i} w_{i}$. Therefore, for any vector field $\mathbf{B}$, the sum

$$
-\mathbf{B} \cdot \nabla \boldsymbol{\beta} \cdot \mathbf{B}-\frac{1}{2} B^{i} B^{j}\left(\boldsymbol{\beta} \cdot \nabla \gamma_{i j}\right)+\frac{1}{2}(\nabla \cdot \boldsymbol{\beta}) B^{i} B_{i},
$$

occurring in (32), equals

$$
\frac{\mathbf{B} \cdot \nabla h}{h} \mathbf{w} \cdot \mathbf{B}-B^{i} B^{j} \partial_{i} w_{j}-\frac{\mathbf{w} \cdot \nabla h}{4 h}|\mathbf{B}|^{2}+\frac{1}{2}|\mathbf{B}|^{2} \operatorname{Divw} .
$$

Since as stated the terms in $\mathbf{w}(\mathbf{x})$ decrease as $\delta(\mathbf{x})^{-1}$, those in $\nabla h, \nabla \mathbf{w}$ like $\delta(\mathbf{x})^{-2}$, and $1 / h(\mathbf{x})=1+O\left(\delta(\mathbf{x})^{-1}\right)$, we may write the term in (B20) in a point $\mathbf{x}$ outside of $\Omega$ as

$$
\left(-B^{i} B^{j} \partial_{i} w_{j}+\frac{1}{2}|\mathbf{B}|^{2} \operatorname{Div} \mathbf{w}\right)(\mathbf{x})+O\left(\delta(\mathbf{x})^{-3}\right)+O\left(\epsilon^{2}\right) .
$$

The first two terms should decay as $\delta(\mathbf{x})^{-2}$. If we consider points at some distance from $\Omega$, we may ignore the remainders as inferior by an order of magnitude.

As for $\alpha^{2}$, from (B11) we find

$$
\alpha^{2}=\frac{|\mathbf{w}|^{2}}{h}-g_{00} .
$$

$g_{00}$ is defined in (B1). $\alpha^{2}$ may be evaluated as follows: let $G$ be the function defined in $\Omega$,

$$
G=2 \rho+4 \rho v^{2}+4 \rho U+2 \rho \Pi+6 p .
$$

Then, with a minor abuse of notation,

$$
\alpha^{2}=\frac{|\mathbf{w}|^{2}}{h}+1+2 U^{2}-\int_{\Omega} \frac{G\left(\mathbf{x}^{\prime}\right)}{\left|\mathbf{x}-\mathbf{x}^{\prime}\right|} d^{3} \mathbf{x}^{\prime} .
$$

Thus

$$
\begin{aligned}
\nabla \alpha^{2}= & -\frac{|\mathbf{w}|^{2} \nabla h}{h^{2}}+\frac{2 \mathbf{w} \cdot \nabla \mathbf{w}}{h}+2 U \nabla U \\
& +\int_{\Omega} \frac{G\left(\mathbf{x}^{\prime}\right)}{\left|\mathbf{x}-\mathbf{x}^{\prime}\right|^{3}}\left(\mathbf{x}-\mathbf{x}^{\prime}\right) d^{3} \mathbf{x}^{\prime} .
\end{aligned}
$$

As before, the first three terms decrease like $\delta(\mathbf{x})^{-3}$, while the fourth one behaves like $\delta(\mathbf{x})^{-2}$. Since

$$
\frac{1}{2 \alpha(\mathbf{x})}=\frac{1}{2}+O\left(\delta(\mathbf{x})^{-2}\right),
$$

we may write

$$
\begin{aligned}
\nabla \alpha(\mathbf{x})= & \frac{1}{2} \int_{\Omega} \frac{G\left(\mathbf{x}^{\prime}\right)}{\left|\mathbf{x}-\mathbf{x}^{\prime}\right|^{3}}\left(\mathbf{x}-\mathbf{x}^{\prime}\right) d^{3} \mathbf{x}^{\prime}+O\left(\delta(\mathbf{x})^{-3}\right) \\
& +O\left(\epsilon^{2}\right) .
\end{aligned}
$$

(B22) and (B28) are our main estimates for analyzing the dynamo effect of the body in $\Omega$. 
[1] C. W. Misner, K. S. Thorne, and J. A. Wheeler, Gravitation (Freeman, San Francisco, 1973).

[2] R. D. Blandford and R. L. Znajek, Mon. Not. R. Astron. Soc. 179, 433 (1977).

[3] D. A. McDonald and K. S. Thorne, Mon. Not. R. Astron. Soc. 198, 345 (1982).

[4] K. S. Thorne and D. A. McDonald, Mon. Not. R. Astron. Soc. 198, 339 (1982).

[5] D. A. Uzdensky, Astrophys. J. 603, 652 (2004).

[6] D. A. Uzdensky, Astrophys. J. 620, 889 (2005).

[7] J. Baker and R. Puzio, Phys. Rev. D 59, 044030 (1999).

[8] S. Dain, Phys. Rev. Lett. 87, 121102 (2001).

[9] M. Lyutikov, Phys. Rev. D 83, 064001 (2011).

[10] C. Palenzuela, L. Lehner, and S. Yoshida, Phys. Rev. D 81, 124023 (2010).

[11] C. Palenzuela, M. Anderson, L. Lehrer, S. L. Liebling, and D. Neilsen, Phys. Rev. Lett. 103, 081101 (2009).

[12] M. Núñez, Phys. Rev. Lett. 79, 796 (1997).

[13] R. Khanna, Mon. Not. R. Astron. Soc. 295, L6 (1998).

[14] T. W. Baumgarte and S. L. Shapiro, Astrophys. J. 585, 921 (2003).

[15] T. W. Baumgarte and S. L. Shapiro, Numerical Relativity: Solving Einstein's Equations of the Computer (Cambridge University Press, Cambridge, 2010).

[16] X.-H. Zhang, Phys. Rev. D 39, 2933 (1989).

[17] L. Del Zanna, O. Zanotti, N. Bucciantini, and P. Londrillo, Astron. Astrophys. 473, 11 (2007).
[18] N. Bucciantini and L. Del Zanna, Astron. Astrophys. 528, A101 (2011).

[19] J. C. McKinney, Mon. Not. R. Astron. Soc. 367, 1797 (2006).

[20] S. Koide, Astrophys. J. 708, 1459 (2010).

[21] S. L. Liebling, L. Lehner, D. Neilsen, and C. Palenzuela, Phys. Rev. D 81, 124023 (2010).

[22] M. Takamoto and T. Inoue, Astrophys. J. 735, 113 (2011).

[23] M. Marklund and C. Clarkson, Mon. Not. R. Astron. Soc. 358, 892 (2005).

[24] D. L. Meier, Astrophys. J. 605, 340 (2004).

[25] C. Palenzuela, L. Lehner, O. Reula, and L. Rezzolla, Mon. Not. R. Astron. Soc. 394, 1727 (2009).

[26] C.M. Will, Theory and Experiment in Gravitational Physics (Cambridge University Press, Cambridge, 1993).

[27] L. Blanchet, G. Faye, and B. Ponsot, Phys. Rev. D 58, 124002 (1998), 1-20.

[28] S. Kopeikin and I. Vlasov, Phys. Rep. 400, 209 (2004).

[29] T. Clifton, Phys. Rev. D 77, 024041 (2008).

[30] S. S. Komissarov, Mon. Not. R. Astron. Soc. 350, 427 (2004).

[31] S. S. Komissarov, Mon. Not. R. Astron. Soc. 418, L94 (2011).

[32] D. Biskamp, Nonlinear Magnetohydrodynamics (Cambridge University Press, Cambridge, 1993).

[33] A. Gilbert and C. S. Childress, Stretch, Twist and Fold: The Fast Dynamo (Springer, New York, 1995). 